\title{
Chimeric Antigen Receptor Engineered T (CAR-T) Cells and Cancer Therapy
}

\author{
*Nahla A M Hamed \\ Professor of Hematology, Alexandria University, Egypt \\ Submission: August 07, 2016; Published: August 31, 2016 \\ "Corresponding author: Nahla A M Hamed, Professor of Hematology, Alexandria University, Egypt.
}

\section{Editorial}

Gene therapy has demonstrated significant potential as a cancer therapy in the last few years. The greatest successes have been reached by genetic modification of autologous patient $\mathrm{T}$ cells with chimeric antigen receptors (CARs), which are novel and synthetic receptors composed of the antigen-binding domain from a B cell receptor fused to the signaling elements associated with a $\mathrm{T}$ cell receptor [1].

CAR-T cells are targeted towards malignant cells expressed defined associated antigens. CAR-T cells can overcome the major challenges of immunotherapy such as tolerance to self-antigens and cancer immune system evasion mechanism [2].

\section{Structure of CAR}

CAR is composed of an extracellular antigen-bindingdomain derived from an antibody (mostly a single-chain fragment of variable region antibody), a hinge region, transmembrane domains and an intracellular signaling chain, frequently the TCR-derived CD3 chain [3].

Most investigators use the hinge and transmembrane domains of CD8 or CD28 [4]. The antibody domain mediates target recognition independently of major histocompatibility complex and enables the targeting of a plethora of antigens, including proteins, carbohydrates and gangliosides, as long as the antigen is present on the surface of the target cell [5].

Retroviruses are used to introduce the CAR constructs into $\mathrm{T}$ cells. One potential disadvantage of retrovirus as vehicle is the potential for silencing of CAR expression based on silencing of the long terminal repeats. This could be an advantage of CARbased therapies if they are used as a bridge to another definitive treatment such as allogeneic bone marrow transplant [4]. Lentiviral vectors are potentially safer than retrovirus based on integration preferences examined in hematopoietic stem cells, though it is not clear that this applies to primary human $\mathrm{T}$ cells. Use of specific promoters in combination with Lentiviral transduction has enabled sustained surface expression of CARs on transduced T cells; this likely extends the survival of functional CAR T cells in vivo [4].

\section{The Generations of CARs}

CARs are described as first-, second- or third generation [6]. The "generations" of CARs typically refer to the intracellular signaling domains they contain. First-generation CARs include only CD3 as an intracellular signaling domain. They lack costimulatory properties. Second-generation CARs include a single costimulatory domain derived from either CD28 or 4-1BB to fully activate $T$ cells. Third-generation CARs include two costimulatory domains, such as CD28, 4-1BB, and other costimulatory molecules in tandem [4]. Preclinical experiments suggest that third generation CARs may be more potent than second generation CARs [7].

Clinical studies of first-generation CAR $\mathrm{T}$ cells showed that lympho depleting chemotherapy may enhance CAR T-cell responses by eradicating regulatory $\mathrm{T}$ cells, eliminating other immune cells that may compete for homeostatic cytokines, and enhancing antigen presenting cell activation [8].

\section{Clinical Studies of CAR T cells}

\section{CAR $T$ cells and hematologic malignancies}

The most investigated target for CARs is CD19 because of its common expression in most B cell leukemias and lymphomas, and its absence in all normal tissues other than B cell lineage [9]. CD19-targeted CAR constructs have demonstrated consistently high antitumor efficacy in children and adults with relapsed B-cell acute lymphoblastic leukemia (B-ALL), chronic lymphocytic leukemia (CLL), and B-cell non-Hodgkin lymphoma (B-NHL) in the non-transplantation setting [8].

ALL: Dramatic results have been reported with use of CAR $\mathrm{T}$ cells in ALL [10]. Anti-CD19 CAR T cells can lead to complete response rates of up to $90 \%$ in heavily pretreated ALL patients. These high response rates are tempered by the requirement for individual product manufacturing for each patient, the high costs of gene transfer technology and emerging problems such as limited persistence in some patients and antigen-loss relapses. Preliminary results indicate that molecules other than CD19 can also be effectively targeted [11]. 


\section{Cancer Therapy \& Oncology International Journal}

a) NHL: Promising results have been seen in NHL patients [10].

b) CLL: Limited clinical efficacy of CART cells was observed in CLL patients compared to B-ALL. Potential explanations is limited persistence of CAR T cells in CLL patients, the immuno-inhibitory tumor microenvironment of CLL, the lymph-node based disease in CLL compared to the mostly bone marrow-based nature of B-ALL and the lower tumor burden at treatment in B-ALL patients. Potential methods to overcome these possible barriers include incorporation of other signaling domains or other immune effectors into the CAR T cells. Limitations of persistence may be overcome by incorporating co-stimulatory domains such as CD28, CD137 or CD134 into third-generation CARs or by directing secretion of pro-inflammatory cytokines such as IL12 in a second-generation CAR [1].

c) Acute myeloid leukemia: A CD33-specific CAR has been developed [12] and is effective in preclinical experiments. However, this approach needs further evaluation asCD33 is a pan-myeloid marker and so, CD33CAR-redirected $\mathrm{T}$ cells may lead to profound and prolonged myeloid depletion. The isoform variant 6 of CD44 (CD44v6) represents another possible target for CAR-T cells in myeloid leukemias. Preliminary results show that CD44v6-CARredirected $\mathrm{T}$ cells had antitumor effects against CD44v6positive malignancies [13].

d) Multiple myeloma (MM): A recent case report described the use of CD19 CAR T cells after a second ASCT in a MM patient. The patient sustained a CR without evidence of recurrence at 12 months [10]. Allogeneic CD19-directed CAR T cells (derived from donor lymphocytes) have induced remissions without induction of GVHD in post allo-HCT relapsed patients. Thus, the allo-HCT or ASCT platform could be adapted to subsequent CAR T technology [14].

Given the extremely low expression of CD19 on the patient's neoplastic plasma cells [10], several promising antigenic targets have been identified for the development of anti-MM CARs such as B-cell maturation antigen, CD138, kappa light chains and CS-1 [14].

\section{CAR-T cell-and minimal residual disease}

CAR-T cell-based therapy may be better suited to minimal residual disease or as an adjuvant for patients at high risk of relapse, who responded to salvage treatment or after transplant [7].

\section{CAR T cells and transplantation}

CAR T cells targeting CD19 have served as a bridge to transplantation or have been used as salvage for patients who relapse or progress after transplantation. Ongoing studies are examining the role of combining these therapies with stem cell transplantation to further improve outcomes in lymphoma and MM patients [10].

\section{CAR T-cell dose}

The cell dose for patients with morphologic disease is lower than those with MRD (1X106vs3 X 106 19-28zCART cells per $\mathrm{kg}$ ). A higher CAR T-cell dose is well tolerated in patients with MRD [8].

\section{Toxicities of CD19-targeted CAR T cells}

All trials of CD19-targeted CAR T cells have reported similar treatment related toxicities, particularly cytokine release syndrome (CRS), neurological toxicities, and B-cell aplasia, although severity of observed toxicities differs. CRS reflects a systemic inflammatory response syndrome hours to days following CAR T-cell infusion, characterized by elevations of proinflammatory cytokines and T-cell activation and expansion [8]. Clinical features include fever, myalgia, malaise, and, in more severe cases, a capillary leak syndrome associated with hypoxia, hypotension, and occasionally renal dysfunction and coagulopathy. Severe CRS may be treated with the IL-6 receptor inhibitor tocilizumab or with lymphotoxic corticosteroids [8].

\section{CAR T cells and solid tumors}

Unfortunately, the clinical results in solid tumors have been much less encouraging. Specific target antigens on solid tumors are more difficult to identify. Roughly 30 solid tumor antigens are being evaluated for CAR T-cell therapy. The two most positive trials reported were GD2 CARs to target neuroblastoma and HER2 CARs for sarcoma [15].

The solid tumor landscape presents unique barriers that are absent in hematological malignancies. Even after successful trafficking and infiltration, $\mathrm{T}$ cells must surmount challenges conferred by: (i) an environment characterized by oxidative stress, nutritional depletion, acidic $\mathrm{pH}$ and hypoxia; (ii) the presence of suppressive soluble factors and cytokines;(iii) suppressive immune cells (regulatory T cells), myeloid derived suppressor cells, tumor-associated macrophages or neutrophils; and (iv) T-cell-intrinsic negative regulatory mechanisms (e.g., upregulation of cytoplasmic and surface inhibitory receptors) and over expression of inhibitory molecules [15].

TRUCKs ( $T$ cells redirected for universal cytokine mediated killing)

TRUCKs are CAR-redirected $\mathrm{T}$ cells used as vehicles to constitutively produce or induce release, mostly a proinflammatory cytokine in the targeted tissue. CAR T cells, when activated by their CAR, deposit IL-12 in the targeted tumor lesion, which in turn attracts an innate immune cell response toward cancer cells that are invisible to CAR T cells [5]. TRUCKs exhibited remarkable efficacy against solid tumors with diverse cancer cell phenotypes, suggesting their evaluation in clinical trials [5]. 


\section{Cancer Therapy \& Oncology International Journal}

\section{References}

1. Davila ML, Bouhassira DCG, Park JH, Curran KJ, Smith EL, et al (2014) Chimeric antigen receptors for the adoptive $\mathrm{T}$ cell therapy of hematologic malignancies. Int J Hematol 99(4): 361-371.

2. Resetca D, Neschadim A, Medin JA (2016) Engineering hematopoietic cells for cancer immunotherapy: strategies to address safety and toxicity concerns. J Immunother 39(7): 249-259.

3. Nakazawa Y (2016) Gene modified T cell therapy using chimeric antigen receptor for pediatric hematologic malignancies. Rincho Ketsueki 57(6): 701-708.

4. Maus MV, Grupp SA, Porter DL, June CH (2014) Antibody-modified T cells: CARs take the front seat for hematologic malignancies. Blood 123(17): 2625-2635.

5. Chmielewski M, Hombach AA, Abken H (2014) Of CARs and TRUCKs chimeric antigen receptor (CAR) T cells engineered with an inducible cytokine to modulate the tumor stroma. Immunological Reviews 257(1): 83-90.

6. Garfall AL, Fraietta JA, Maus MV (2014) Immunotherapy with chimeric antigen receptors for multiple myeloma. Discov Med 17(91): 37-46.

7. Hoyos V, Savoldo B, Dotti G (2012) Genetic modification of human $\mathrm{T}$ lymphocytes for the treatment of hematological malignancies. Haematologica 97(11): 1622-1631.

8. Park JH, Geyer MB, Brentjens RJ (2016) CD19-targeted CAR T-cell therapeutics for hematologic malignancies: interpreting clinical outcomes to date. Blood 127(26): 3312-3320.
9. van der Stegen SJC, Hamieh M, Sadelain M (2015) The pharmacology of second-generation chimeric antigen receptors. Nature Reviews 14: 499-509.

10. Perales MA, Sauter CS, Arman P (2016) Fast Cars and no brakes: autologous stem cell transplantation as a platform for novel immunotherapies. Biol Blood Marrow Transplant 22: 17-22.

11. Planes GS (2016) Trains, and automobiles: Perspectives on CAR T cells and other cellular therapies for hematologic malignancies. Curr Hematol Malig Rep 11(4): 318-325.

12. Marin V, Pizzitola I, Agostoni V, Attianese GM, Finney H, et al. (2010) Cytokine-induced killer cells for cell therapy of acute myeloid leukemia: improvement of their immune activity by expression of CD33-specific chimeric receptors. Haematologica 95(12): 2144-2152.

13. Casucci M, Falcone L, Nicolis di Robilant B, Camisa B, Genovese P et al. (2011) Dual transgenesis of T cells with a novel CD44v6-specific chimeric antigen receptor and a suicide gene for the safe and effective targeting of chemoresistance in hematopoietic tumors. Blood 118: 3125 .

14. Krishnan A, Vij R, Keller J, Dhakal B, Hari P (2016) Moving beyond autologous transplantation in multiple myeloma: consolidation, maintenance, allogeneic transplant, and immune therapy. Asco Educational Book 35: 210-221.

15. Newick K, Moon E, Albelda SM (2016) Chimeric antigen receptor T-cell therapy for solid tumors. Molecular Therapy - Oncolytics 3: 16006. 